\title{
A Wideband Contactless and Bondwire-Free MMIC to Waveguide Transition
}

\author{
Alhassan Aljarosha, Ashraf Uz Zaman, Member, IEEE, and Rob Maaskant, Senior Member, IEEE
}

\begin{abstract}
A contactless transition from a high-permittivity microstrip line $\left(\epsilon_{\mathbf{r}}=9.9\right)$ to an air-filled waveguide (WG) has been impedance-matched over a large simulated relative bandwidth of $38 \%$ (W-band, 75-110 GHz). The transition couples the EM fields directly from the MMIC's microstrip line via an SIW and an off-chip stub section to a ridge WG section. The novel structure is low loss and suits pick-and-place assembly techniques of mm-Wave MMICs inside metal WGs. The design process is detailed and manufacturing tolerances of the Alumina prototype $P C B$ are discussed. The measured back-to-back structure retains an appreciable insertion loss smaller than $0.8 \mathrm{~dB}$ for a single transition and a fractional bandwidth of $28 \%(72-95 \mathrm{GHz})$ over which the return loss is greater than $10 \mathrm{~dB}$.
\end{abstract}

Keywords-Waveguide transition, MMICs, contactless connection, integration.

\section{INTRODUCTION}

$\mathrm{T}$ realize next generation wireless systems cost effective manufacturing technologies and high-performance lowloss transitions between waveguides (WGs) and integrated circuits (MMICs) need to be developed. Galvanic contactless transitions between printed circuit boards (PCBs) and WGs are advantageous in this respect, particularly at $\mathrm{mm}$-wave frequencies. Moreover, electromangetic (EM) coupling directly from MMICs to WGs obviate the use of (inductive) RFbondwire or flip-chip based interconnects, thereby reducing the loss and increasing the bandwidth. Achieving wideband impedance matching for direct transitions is a challenge due to the high permittivity contrast between the MMIC's bulk substrate and the air-filled WG. Also, RF power leakage of inline transitions is challenging for $\mathrm{H}$-plane split WGs, however, they can host relatively wide MMICs, as opposed to E-plane split WGs where an oversized WG probe opening can leak higher-order modes. This paper proposes a solution to these challenges for the H-plane split WG case.

Microstrip line to WG transitions have been heavily researched. Among them, a solder-joint-free inline microstrip to conventional WG transition [1], offering $S_{11}<-10 \mathrm{~dB}$ and $S_{21}>-1.1 \mathrm{~dB}$, from $8.5-9.5 \mathrm{GHz}(11 \%$ measured rel. bandwidth), albeit for a relatively low $\epsilon_{\mathrm{r}}=3.8$ substrate. A

Manuscript received Oct. 11, 2016; revised Jan. 02, 2017; accepted Feb. 15, 2017. Date of publication Jun. XX, 2017; date of current version Jul. XX, 20XX. This work was supported by the European and Swedish Research Councils: VR grant ID 621-2011-4651: an advanced investigator Grant (no. 321125) and the VINNOVA Chase Research Center. The authors are with the Department of Signals and Systems, Chalmers Univ. of Techn., S-41296 Gothenburg, Sweden. e-mail: alhalj@ student.chalmers.se, rob.maaskant@chalmers.se. Rob Maaskant is also with the electromagnetics group of the Eindhoven University of Technology (TU/e). Color versions of one or more of the figures in this letter are available online at http://ieeexplore.ieee.org.

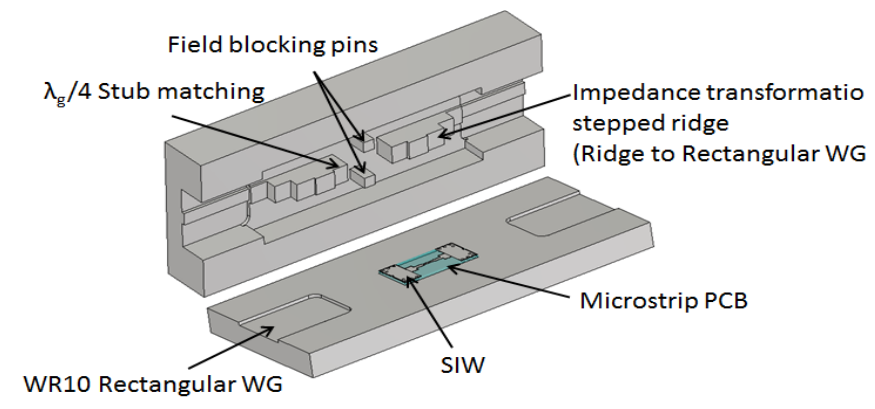

Fig. 1. The proposed microstrip-to-WG transition

similar transition has shown to cover the entire W-band in simulations only, with $S_{11}<-15 \mathrm{~dB} ; S_{21}>-0.55 \mathrm{~dB}$, for $\epsilon_{\mathrm{r}}=2.2$ [2]. Still, a bondwire or flip-chip design is needed to interface a high-permittivity MMIC to the low permittivity application board featuring the transition. The E-plane probe design in [3] covers the entire W-band, but also employs a low-permittivity substrate $\left(\epsilon_{\mathrm{r}}=2.2\right)$ and requires cutting of the substrate which is undesired in case of direct MMIC transitions. The $90^{\circ}$ radial probe in [4] has a $45 \%$ fractional$\mathrm{BW}$, but it needs a $\lambda / 4$ back-short and is an E-plane split-block solution. The H-plane split block SIW-to-WG direct transition in [5] $\left(\epsilon_{\mathrm{r}}=3\right)$ exhibits an $S_{11}<-15 \mathrm{~dB} ; S_{21}>-0.68 \mathrm{~dB}$ (back-to-back). Although it operates at the lower frequency of $40 \mathrm{GHz}$, it is capable of offering a rel. bandwidth of $45 \%$.

Our design not only covers the entire W-band (in simulations), but also couples the RF fields directly from a highpermittivity substrate - representing the chip's bulk substrate - to the principal rectangular WG mode. Bonding is only needed for DC-biasing. The prototype chip is manufactured by ATCeramics as an 'ordinairy' alumina PCB of chip size $1.5 \times 3.0 \mathrm{~mm}^{2}\left(\epsilon_{\mathrm{r}}=9.9\right)$. The mechanical tolerances of these tiny PCBs are discussed. The electrical properties of Alumina are not specified by the vendor at around $100 \mathrm{GHz}$. Despite these tolerances and uncertainties, the manufactured prototype transition features a $28 \%$ measured rel. bandwidth (72-95.5 GHz) and an average insertion loss of $1.4 \mathrm{~dB}$ (backto-back). It is expected that the PCB and WG parts in Fig. 1 can be assembled in a fast and easy manner through pick-andplace techniques.

The paper is organized as follows. Sec. II presents the design steps and operation principle of the back-to-back (B2B) structure; Sec. III shows the experimental validation of the transition; Sec. IV examines causes for the performance reduction of the measured prototype, and; Sec. V contains the conclusions. 


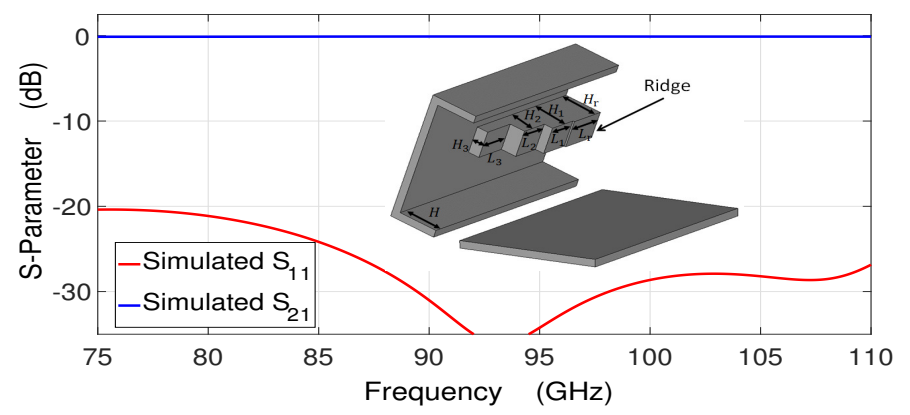

Fig. 2. Impedance-matched rectangular to ridge WG transition and its simulated S-parameters. Optimized design parameters: $H=1 \mathrm{~mm} ; H_{\mathrm{r}}=$ $0.96 \mathrm{~mm} ; H_{1}=0.92 \mathrm{~mm} ; H_{2}=0.69 \mathrm{~mm} ; H_{3}=0.29 \mathrm{~mm}$; $L_{1}=0.79 \mathrm{~mm} ; L_{2}=0.76 \mathrm{~mm}$, and; $L_{3}=0.87 \mathrm{~mm}$.

\section{Stepwise Design of Proposed Transition}

The commercially available CST Microwave Studioß 2013 is used for the design of the rectangular WG to microstrip line transition (-35 $\mathrm{dB}$ rel. accuracy level; time domain solver). A single transition consists of three parts:

1) Rectangular to Ridge WG Transition (Fig. 2): A standard three-step Chebyshev transformer based on $\lambda_{\mathrm{g}} / 4$ sections impedance matches the rectangular $\mathrm{WG}$ to the ridge $\mathrm{WG}$. The final optimized design parameters are shown in Fig. 2. Standardized waveguide ports are used on either side. The simulation result shows a wideband performance over the entire W-band $\left(S_{11}<-15 \mathrm{~dB}, 75-110 \mathrm{GHz}\right)$.

2) Novel Ridge WG to SIW Contactless Transition (Fig. 3): The ridge is extended by an open-ended $\lambda_{\mathrm{g}} / 4=0.617$ $\mathrm{mm}$ stub slightly placed above the upper SIW ground plane [cf. Fig. 3(a)], effectively providing a short-circuit for the fields traveling inside the air gap of the ridge waveguide into the substrate of the PCB's SIW [cf. Fig. 3(b)]. Note how the upper ground plane of the PCB shields the interior SIW fields from the stub fields. Two inductive tuning vias and a U-shaped notch partly compensate for the stub capacitance, thereby increasing the operational bandwidth (dashed vs. solid lines in Fig. 3, also showing SIW geometrical dimensions). The one mil necessary pullback avoids burrs in the metalization when dicing the substrate, but has negligible effect on the EM performance.

3) SIW to Microstrip Line Transition (Fig. 4): The SIW is impedance-matched to a $50 \Omega$ microstrip line via standard steptapered microstrip line sections. The optimized geometrical dimensions and computed B2B S-parameters are shown in Fig. 4. Both the SIW and microstrip line are on an alumina substrate $\left(\epsilon_{r}=9.9\right)$ of thickness $h=0.1 \mathrm{~mm}$. The high permittivity is comparable to typically employed undoped/intrinsic MMIC bulk substrates at mm-wave frequencies. The transition can also be designed for thinner substrates (e.g. $50 \mu \mathrm{m}$ ).

\section{B2B PRototype And Measurement Results}

Based on the above steps, the B2B prototype transition in Fig. 1 was designed, manufactured, and validated through measurements. The field blocking pins in Fig. 1 increase the isolation of the fields between the open-ended stubs @ $84 \mathrm{GHz}$ in the hollow cavity above the PCB (cavity-mode suppression

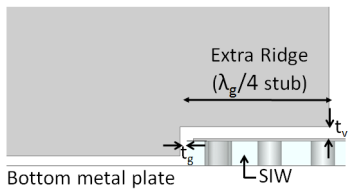

(a)

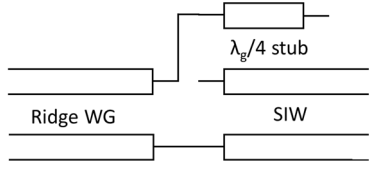

(b)

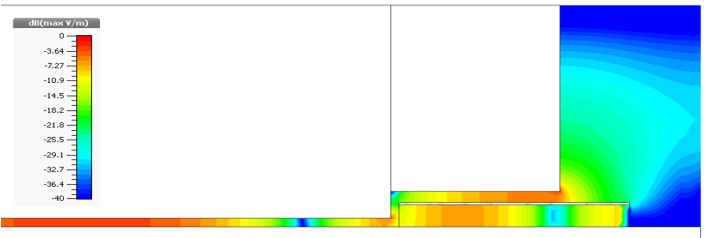

(c)

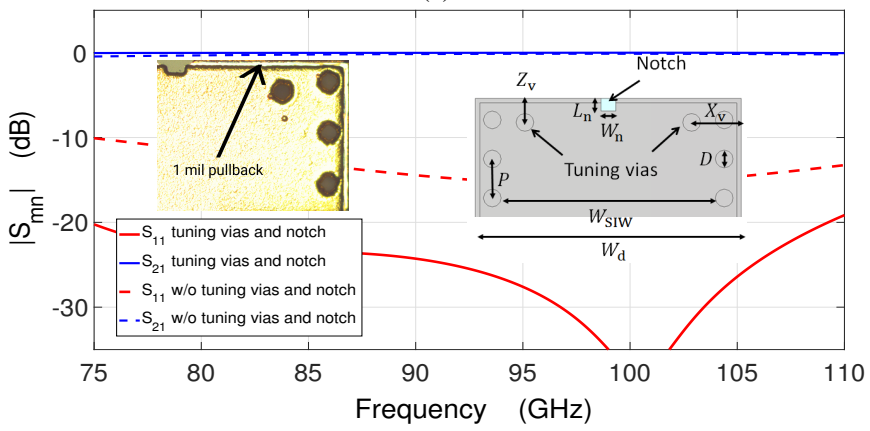

(d)

Fig. 3. (a) Side view of ridge WG to SIW contactless transition (WG side walls are hidden): $t_{\mathrm{v}}=0.048 \mathrm{~mm} ; t_{\mathrm{g}}=0.03 \mathrm{~mm}$; (b) Single mode transmission line model for understanding the first-order EM coupling mechanism of the contactless transition; (c) The real part of the total Efield distribution in dBmax $\mathrm{V} / \mathrm{m}$; (d) SIW geometrical dimensions: $Z_{\mathrm{v}}=$ $0.135 \mathrm{~mm} ; L_{\mathrm{n}}=0.071 \mathrm{~mm} ; W_{\mathrm{n}}=0.083 \mathrm{~mm} ; X_{\mathrm{v}}=0.28 \mathrm{~mm}$; $D=0.1 \mathrm{~mm} ; P=0.22 \mathrm{~mm} ; W_{\text {SIW }}=1.2 \mathrm{~mm} ; W_{\mathrm{d}}=1.5 \mathrm{~mm}$; and microscopic close up of the PCB.

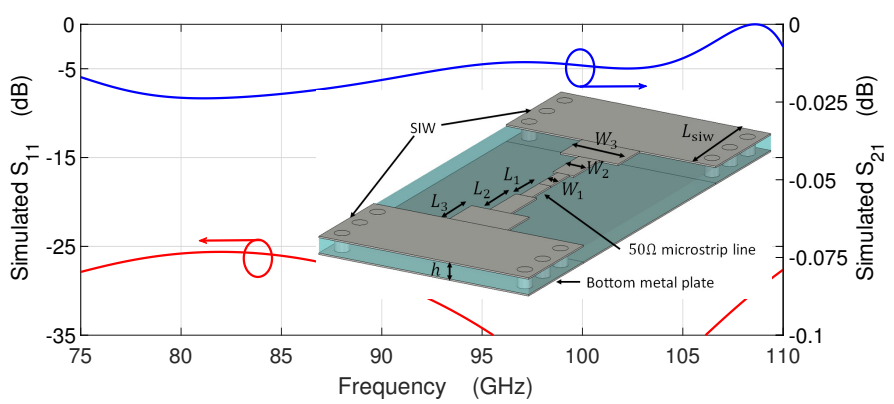

Fig. 4. SIW to microstrip line transition: $W_{1}=0.085 \mathrm{~mm} ; W_{2}=$ $0.148 \mathrm{~mm} ; W_{3}=0.41 \mathrm{~mm} ; L_{1}=0.23 \mathrm{~mm} ; L_{2}=0.32 \mathrm{~mm}$; $L_{3}=0.27 \mathrm{~mm} ; L_{\mathrm{SIW}}=0.68 \mathrm{~mm}$; and simulated S-parameters for a B2B configuration.

results not shown), which is particularly important for active MMICs when high input-output isolation is required. Two gap waveguide pins are used for this purpose [6], of height $\lambda / 4=0.81 \mathrm{~mm}$ and width $0.43 \mathrm{~mm}$, each of which is centered in the middle of a long PCB edge. Furthermore, the following solver settings are used: 38 lines per wavelength yielding 4,638,128 mesh cells. An Agilent PNA Network Analyzer 

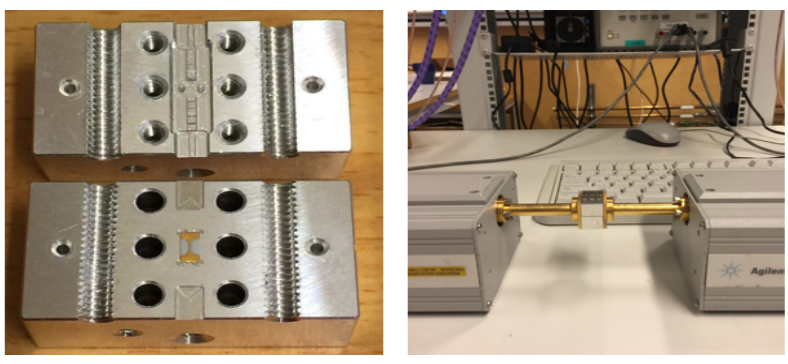

Fig. 5. Prototype of B2B transition and VNA measurement setup

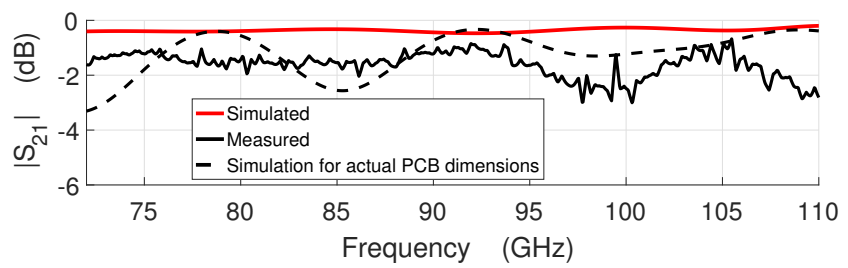

(a)

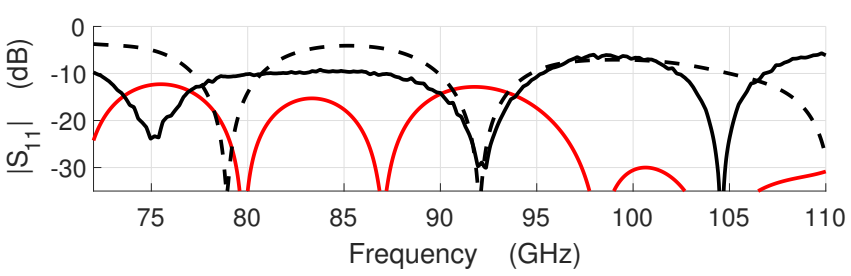

(b)

Fig. 6. Simulated and measured S-parameters for the B2B transition.

(N5222A, 10-26.5 MHz) connected to N5260-6003 extender modules have been used to measure the prototype in the 67-110 GHz frequency band. Standard WR10 WG flanges interface the CNC-milled aluminium prototype as illustrated in the measurement setup in Fig. 5.

The simulated return loss (RL) in Fig. 6 is better than $15 \mathrm{~dB}$ over the entire W-band ( $>38 \%$ rel. bandwidth), while the insertion loss (IL) is less than $0.18 \mathrm{~dB}$ (PEC material). Furthermore, due to mechanical tolerances and material uncertainties ( $c f$. next section), the measured RL reduces to about $10 \mathrm{~dB}$ in the 72-95.5 GHz range, while the corresponding IL is on average $1.4 \mathrm{~dB}$, thus retaining an appreciable $28 \%$ rel. bandwidth. Nonetheless, causes for this performance reduction have been studied whose findings are summarized below.

\section{TOLERANCE ANALYSiS}

The milling measurement report of ASTRON's Mechanical Production Lab concludes that: "All measured dimensions are within 5-10 micron to the nominal required value, excluding the measurement uncertainty of about 5 micron". These milling tolerances are well within the anticipated $20 \mu \mathrm{m}$ tolerance range as determined by our simulations. Further examination using both optical and mechanical microscopic measurement techniques revealed that the metallization thickness of the manufactured PCB is $16 \pm 1 \mu \mathrm{m}$, instead of the designed
$10 \mu \mathrm{m}$, and that the substrate thickness is about $112 \pm 4 \mu \mathrm{m}$, instead of $4 \mathrm{mil}(\sim 102 \mu \mathrm{m})$. The extra thickness of both the metallization and the substrate affect the impedance of the stub (i.e. air gap between stub and PCB reduces) as well as the microstrip lines. The EM simulation results of the $\mathrm{B} 2 \mathrm{~B}$ structure hosting the $\mathrm{PCB}$ of actual dimensions are also overlayed in Fig. 6 (dashed line). The agreement between simulations and measurements clearly improve. The complexvalued permittivity of Alumina remains an uncertain factor as this value is not specified by ATCeramics at $\sim 100 \mathrm{GHz}$. Finally, the PCB placement error has little effect on the simulated results, i.e., $\pm 10 \mu \mathrm{m}$ displacement results in $\sim 3 \mathrm{~dB}$ variation at $20 \mathrm{~dB} S_{11}$-level.

\section{Conclusions}

A novel W-band $(75-110 \mathrm{GHz})$ contactless microstrip to rectangular WG transition has been designed, manufactured, and tested. A key feature of the structure is that the $T E_{10}$ ridge waveguide mode is coupled to the principal SIW substrate mode with the help of a tuning stub that partially overlaps the PCB. Another feature is that the PCB employs a highpermittivity substrate rendering the use of MMIC bondwire or flipchip designs unnecessary (for RF signals). Although the entire W-band is covered in simulations, due to mechanical tolerances and uncertainties in material properties, the measured return loss remains good $(>10 \mathrm{~dB})$ over a $28 \%$ rel. bandwidth.

The herein proposed transition allows easy integration of $\mathrm{RF}$ electronics in WG technology by means of fast, flexible, and low-cost pick-and-place assembly techniques employing low-loss contactless interconnects at mm- and sub-mm wave frequencies. Based on this passive transition, the next milestone is therefore to truly integrate an active component inside a waveguide through contactless interconnects.

\section{ACKNOWLEDGMENT}

Thanks to Ir. J. Pragt and M. Schuil (ASTRON, CNC milling); and S. Rahiminejad (Chalmers, opt. measurements).

\section{REFERENCES}

[1] Y. Zhang, J. Ruiz-Cruz, K. Zaki, and A. Piloto, "A waveguide to microstrip inline transition with very simple modular assembly," IEEE Microw. Wireless Compon. Lett., vol. 20, no. 9, pp. 480-482, Sep. 2010.

[2] A. Rebollo, B. Larumbe-Gonzalo, R. Gonzalo, and I. Ederra, "Full Wband microstrip-to-waveguide inline transition," in The $8^{r t} h$ European Conference on Antennas and Propagation (EuCAP 2014), 2010, pp. 2591-2593.

[3] E. S. Li, G. X. Tong, and D. C. Niu, "Full W-band waveguide-tomicrostrip transition with new E-plane probe," IEEE Microw. Wireless Compon. Lett., vol. 23, no. 1, pp. 4-6, 2013.

[4] J. W. Kooi, G. Chattopadhyay, S. Withington, F. Rice, J. Zmuidzinas, C. Walker, and G. Yassin, "A full-height waveguide to thin-film microstrip transition with exceptional rf bandwidth and coupling efficiency," International Journal of Infrared and Millimeter Waves, vol. 24, no. 3, pp. 261-284, 2003.

[5] J. L. Cano, A. Mediavilla, and A. R. Perez, "Full-band air-filled waveguide-to-substrate integrated waveguide (SIW) direct transition," IEEE Microw. Wireless Compon. Lett., vol. 25, no. 2, pp. 79-81, 2015.

[6] J. Zhang, X. Zhang, D. Shen, T. Liu, and K. Wu, "Gap waveguidebased PMC packaging for via holes-caused nonsmooth pec surface," IEEE Trans. Compon. Packag. Technol., vol. 5, no. 12, pp. 1828-1838, 2015. 